\title{
Sarcoidosis reactivation with immune checkpoint inhibitors
}

\author{
Creusot $Q^{1}$, Pamart $G^{1}$, Mennecier $B^{1}$, Matau $C^{1}$, Robert $C^{2}$, Mascaux $C^{1,3 *}$ and Quoix $E^{1}$ \\ ${ }^{1}$ Department of Pulmonology, Strasbourg University Hospital, 67091 Strasbourg Cedex, France \\ ${ }^{2}$ Département de médecine, Gustave Roussy, Villejuif, et Université Paris-Saclay, Orsay, France \\ ${ }^{3}$ INSERM IRFAC UMR_S1113, Université de Strasbourg, 67200 Strasbourg, France
}

Immune checkpoint inhibitors (ICI) have been quite a dramatic change in cancer therapy especially for melanoma and non-small cell lung cancer $[1,2]$. Checkpoint inhibitors induce a reactivation of the immune system allowing for destruction of malignant cells. They are monoclonal antibodies targeting cytotoxic $\mathrm{T}$ lymphocyte-associated antigen-4 (CTLA-4), programmed cell death protein (PD-1) or programmed cell death ligand (PD-L1). Due to this mechanism of action, immune-related side effects are commonly observed and most of the auto-immune diseases are potential contra-indications to this immunotherapy.

Sarcoidosis is a systemic granulomatosis that may affect several organs but mainly lungs and intrathoracic lymph nodes. It is characterized by the presence of non-caseating granulomas due to continuous inflammation with accumulation of activated CD4 lymphocytes and macrophages. Although the etiology of sarcoidosis remains unknown, this disease is probably the result of an exaggerated immune response to an undefined antigen, possibly infectious, associated with genetic predisposition [3].

A sarcoid-like or a real sarcoidosis may be observed with ICIs. Clinical and radiological manifestations of sarcoid-like or real sarcoidosis (which are similar in their presentation) may induce some difficulties regarding the interpretation of treatment efficacy.

\section{Clinical case}

A woman was first referred in 2002 at the age of 46 years, for a left breast carcinoma classified as T3N1M0, SBRIII. She was treated with induction chemotherapy consisting of 2 cycles of FEC (5FU, Epirubicin and cyclophosphamide) followed by 4 cycles of docetaxel and vinorelbine. A mastectomy with left axillary lymph node dissection $(5 \mathrm{~N}+/ 24)$ was performed and she received then 3 adjuvant cycles with docetaxel and vinorelbine, radiation therapy and hormonotherapy (Tamoxifen then Raloxifen folloxed by Arimidex).

She developed some therapeutic sequelae: pulmonary fibrosis of the left upper lobe and peripheral neuropathy of the lower limbs. In 2015, she was referred to our department for the exploration of thoracic radiological abnormalities on a follow-up CT-scan. There were important bilateral mediastinal and hilar lymph nodes as well as multiple subpleural and perilymphatic micronodules (besides some fibrosis in the posterior part of the left upper lobe). These abnormalities were highly suggestive of the diagnosis of a sarcoidosis, stage 2 , which was histologically confirmed by an EBUS with biopsy of lymph nodes $4 \mathrm{R}$ and 7 . In the absence of symptoms, of abnormalities of pulmonary function tests and of extra-thoracic localisations, a therapeutic abstention was decided and consecutive CT-scans showed spontaneous regression of the lymph nodes and the micronodules.

In 2017, a surgical resection of a cutaneous lesion of the left hip was performed with the diagnosis of extensive superficial melanoma (Breslow thickness $0.45 \mathrm{~mm}$ ). There was no BRAF mutation, but an exon 3 NRAS mutation. The clinical workup (CT-scan and Pet-scan) showed bilateral inguinal and left external iliac lymph nodes, and multiple liver and splenic metastases. A surgical dissection of left inguinal lymph nodes was performed confirming metastases of the melanoma. The patient was enrolled in a clinical trial (NCT02089685) and received a treatment with an anti-PD-1 (pembrolizumab) $200 \mathrm{mg}$ iv every 3 weeks + an anti-CTLA-4 (ipilimumab) $50 \mathrm{mg}$ iv every 6 weeks for four 6-week cycles initiated in January 2018. A durable partial response was obtained with a disappearance of all metastases except two lymph nodes: in the right inguinal and iliac aeras measuring $1.5 \mathrm{~cm}$ and $1 \mathrm{~cm}$ respectively.

A CT-scan performed in September 2018 showed, in comparison to the one performed in 2017 (Figure 1), a slight increase in the size of a right hilar lymph node, subpleural micronodules in the posterior segment of the right upper lobe and in the anterior segment of left upper lobe. There was also a miliary pattern in the inferior part of both upper lobes with subpleural locations adjacent to the fissures. A bronchofibroscopy was performed with several biopsies of the bronchial spurs which confirmed a granuomatous giganto-cellular inflammation in favor of a sarcoidosis (or sarcoid-like) relapse. The bronchiolaralveolar lavage cellular analysis showed $37 \%$ of lymphocytes, mostly TCD4+ with a ratio TCD4+/TCD8+ of 3.

In the absence of any respiratory function abnormality, we decided with our dermatologist colleagues not to treat sarcoidosis and to continue the ICIs with simple follow-up (Figure 2). Finally, ICIs were stopped after 22 months of treatment in November 2019. In a CT-scan performed in May 2019 while the patient was still under treatment with ICIs, and in April 2020 after stopping ICIs, there was a spontaneous regression of all the abnormalities related to sarcoïdosis. There was also a persistence of the partial response with only one residual hypermetabolic inguinal lymph node.

*Correspondence to: Céline Mascaux, Department of Pulmonology, Strasbourg University Hospital, 67091 Strasbourg Cedex, France, E-mail: celine.mascaux@ chru-strasbourg.fr

Received: October 20, 2020; Accepted: October 30, 2020; Published: November 05, 2020 


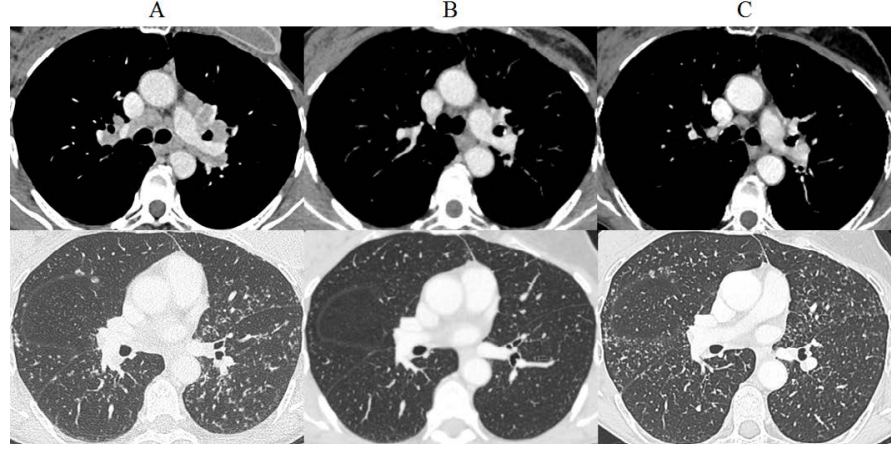

Figure 1. Injected thoracic CT-scan axial with mediastinal and parenchymal windows. (A) 2015 Mediastinal and hilar enlarged lymph nodes, subpleural and parenchyma micronodules (B) 2017 Régression of lymphadenopathies and of the micronodules (C) 2018 Reactivation of the subpleural and parenchymal micronodules (but not of lymph nodes)

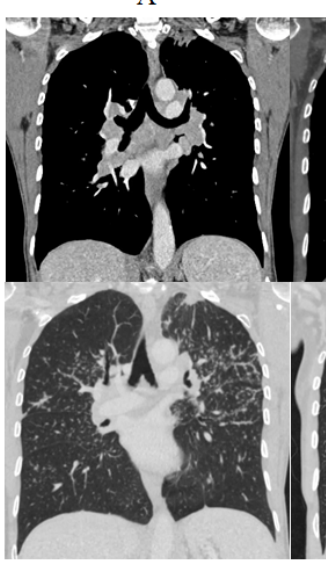

B

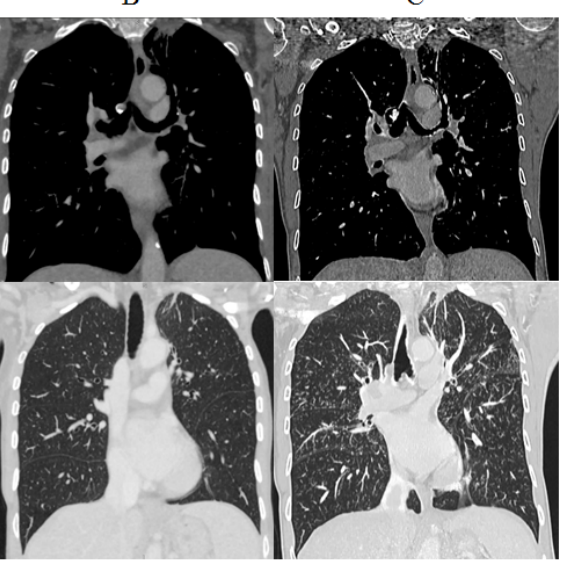

Figure 2. Injected thoracic CT-scan with mediastinal and parenchymal windows (coronal slices). Fibrosis of the apical segment of left upper lobe. (A) 2015 Mediastinal and hilar enlarged lymph nodes, subpleural and parenchymal micronodules (B) 2017 Régression of lymphadenopathies and of the micronodules (C) 2018 Reactivation of the subpleural and parenchymal micronodules (but not of lymph nodes)

\section{Discussion}

This clinical case illustrates two features: first, the development of a sarcoidosis or a sarcoid-like lesion a few years after the diagnosis and the treatment of a breast cancer and second, the reactivation of this sarcoidosis or sarcoid like disease under ICIs. The occurrence of a sarcoidosis before, concurrently or after the diagnosis of a breast cancer is well-known even if it is a rare event $[4,5]$. Although the usual age of occurrence of a sacoidosis is between 20 and 49 years, it develops later on, around 60 years of age, when associated with breast cancer. Sarcoidlike lesions may be due to some drugs besides ICIs, such as interferon alpha, anti-retroviral drugs and anti-TNF- $\alpha$ [6]. The physiopathology of sarcoidosis or sarcoid-like lesion with ICIs is not very clear. ICIs may potentiate the Thelper-1 response, the role of which is of importance in the development of sarcoidosis. In the literature, most of publications are about occurence of sarcoidosis de novo. A clinical case of a patient treated for a bronchial adenocarcinoma with ipilimumab and nivolumab was published in 2016 [7]. At this time, the authors of this case report retrieved 11 previous published cases (10 melanomas et 1 prostatic cancer) among which, 9 treated with ipilimumab alone, 1 with a combination of ipilimumab and nivolumab and one with anti-PDL 1. Sarcoidosis features occurred between the second and the 10th cycle of immunotherapy with a preferential thoracic location the second being cutaneous. Since this publication, 23 other cases have been reported in 2018 [8] and the proportion of lung cancers increased in parallel with the use of ICIs in this setting. As an example a stage 1 sarcoidosis (with mediastinal and hilar lymph nodes) has been described in a 74 years old man who was treated by the combination of carboplatine + pemetrexed + pembrolizumab [9].

Sarcoidosis reactivations are very rarely described in the literature. A case of reactivation has been described in a patient treated with ipilimumab for a melanoma and this sarcoidosis was accompanied by a hypercalcemia [10]. In another case, a patient with a history of thoracic sarcoidosis, stage 1 developed 12 years later, a refractory stage IV Hodgkin disease and was treated with pembrolizumab [11]. Six months after, she developed subcutaneous nodules on her arms. Punch biopsy allowed for the diagnosis of sarcoidosis. She then developed acute iritis of the left eye and bilateral hilar and mediastinal lymph nodes with, on Pet-scan multiple hypermetabolic areas in the bones. All these symptoms disappeared with corticosteroids showing that sarcoidosis was the culprit (and not a Hodgkin relapse). Reactivations are possibly more numerous as quite a lot of sarcoidosis are asymptomatic and thus not diagnosed. Their occurrence at time of treatment with ICIs could sometimes, falsely, suggest a de novo phenomenon. Generally speaking, flare of autoimmune diseases or of close diseases such as sarcoidosis is observed in about $50 \%$ of patients with melanoma [10].

One must keep in mind this adverse event during immunotherapy, as the radiological appearance may be confusing and account for a pulmonary and/or lymphatic relapse of the tumour [11]. It is thus of utmost importance to discuss this differential diagnosis in order not to conclude to the inefficacy of immunotherapy and interrupt the treatment. Moreover, it seems that a sarcoidosis reactivation may be associated with a clinical response of the cancer $[8,10]$. A biopsy should be performed when there are new imaging abnormalities during followup with improvements in some lesions and apparent progression of others. Without a biopsy, it is also impossible to distinguish between sarcoidosis mediastinal lymph nodes and simple reaction lymph nodes [8].

Contrarily to some autoimmune diseases, previous history of sarcoidosis is not a contra-indication to the use of ICIs. In our case, as in the majority of published reactivations of sarcoidosis, the ICIs were not interrupted. Most of time, there is a stabilization of the sarcoidosis [12] or a regression either with corticosteroids $[6,10,11]$ or spontaneously $[9,12,13]$.

As a conclusion, sarcoidosis (or sarcoid-like lesions) is not rare during treatment with ICIs. Sarcoïdosis may occur de novo or be a reactivation of an ancient sarcoidosis. The radiological presentation may be, as in our case, the same as in the first occurrence but may also be different (for example, subcutaneous lesions). In most cases, there is no need to interrupt the ICIs.

\section{References}

1. Hodi FS, O'Day SJ, McDermott DF, Weber RW, Sosman JA, et al. (2010) Improved survival with ipilimumab in patients with metastatic melanoma. $N$ Engl J Med 363 : 711-723. [Crossref]

2. Anagnostou VK, Brahmer JR (2015) Cancer Immunotherapy: A future paradigm shift in the treatment of non-small cell lung cancer. Clin Cancer Res 21: 976-984.

3. Baughman RP, Culver DA, Judson MA (2011) A concise review of sarcoidosis. AJRCCM 183: 573-581.

4. Chen J, Carter III R, Maoz D, Tobar A, Sharon E (2015) Breast cancer and sarcoidosis Case series and review of the literature. Breast Care 10: 137-40. [Crossref] 
5. Schweitzer MD, Salamo O, Holt G, Donna E, Mirsaeidi M (2017) Sarcoidosis onset after breast cancer: A potential association. Eur J Intern Med 44: e11-e12.

6. Chopra A, Nautiyal A, Kalkanis A, Judson MA (2018) Drug-Induced sarcoidosis-like reactions. Chest 154: 664-677.

7. Suozzi KC, Stahl M, Ko CJ, Chiang A, Gettinger SN, et al. (2016) Immune-related sarcoidosis observed in combination ipilimumab and nivolumab therapy. JAAD Case Rep 2: 264-268.

8. Gkiozos I, Kopitopoulou A, Kalkanis A, Vamvakaris IN, Judson MA(2018) Sarcoidosislike reactions induced by checkpoint inhibitors. J Thorac Oncol 13: 1076-1082.

9. Fakhri G, Akel R, Salem Z, Tawil A, Tfayli A (2017) Pulmonary sarcoidosis activation following neoadjuvant pembrolizumab plus chemotherapy combination therapy in a patient with non-small cell lung cancer: A case report. Case Rep Oncol 10: 1070-1075.
10. Johnson DB, Sullivan RJ, Ott PA, Carlino MS, Khushalani NI, et al. (2016) Ipilimumab therapy in patients with advanced melanoma and preexisting autoimmune disorders. JAMA Oncol 2: 234

11. Cotliar J, Querfeld C, Boswell WJ, Raja N, Raz D (2016) Pembrolizumab-associated sarcoidosis. JAAD Case Rep 2: 290-293.

12. Reuss JE, Kunk PR, Stowman AM, Gru AA, Slingluff CL (2016) Sarcoidosis in the setting of combination ipilimumab and nivolumab immunotherapy: A case report \& review of the literature. J Immunother Cancer 4: 94.

13. Danlos FX, Pagès C, Baroudjian B, Vercellino L, Battistella M, et al. (2016) Nivolumabinduced sarcoid-like granulomatous reaction in a patient with advanced melanoma. Chest 149: e133-e136. [Crossref]

Copyright: (C2020 Creusot Q. This is an open-access article distributed under the terms of the Creative Commons Attribution License, which permits unrestricted use, distribution, and reproduction in any medium, provided the original author and source are credited. 